\title{
Engineering membrane and cell-wall programs for tolerance to toxic chemicals: beyond solo genes
}

\author{
NR Sandoval and ET Papoutsakis
}

\begin{abstract}
Metabolite toxicity in microbes, particularly at the membrane, remains a bottleneck in the production of fuels and chemicals. Under chemical stress, native adaptation mechanisms combat hyper-fluidization by modifying the phospholipids in the membrane. Recent work in fluxomics reveals the mechanism of how membrane damage negatively affects energy metabolism while lipidomic and transcriptomic analyses show that strains evolved to be tolerant maintain membrane fluidity under stress through a variety of mechanisms such as incorporation of cyclopropanated fatty acids, trans-unsaturated fatty acids, and upregulation of cell wall biosynthesis genes. Engineered strains with modifications made in the biosynthesis of fatty acids, peptidoglycan, and lipopolysaccharide have shown increased tolerance to exogenous stress as well as increased production of desired metabolites of industrial importance. We review recent advances in elucidation of mechanisms or toxicity and tolerance as well as efforts to engineer the bacterial membrane and cell wall.
\end{abstract}


Introduction - Microbial metabolite toxicity remains a limiting bottleneck in the production of fuels and chemicals

The repertoire of metabolites that can be produced as commodity or specialty chemicals and biofuels or biofuel precursors through microbial processes is ever expanding, and includes organic acids, alkanes, long-chain alcohols and aldehydes, and free fatty acids [1]. Most industrially attractive chemicals are toxic to microbes, an issue that limits the development of economically feasible bioprocesses [1,2]. Engineering or adapting organisms to tolerate high titers of toxic chemicals under realistic metabolite-production conditions aim to make them capable of producing toxic metabolites faster and at higher titers. Thus, understanding and developing microbial tolerance to a broad spectrum of chemicals is a problem of major industrial significance [1]. It is necessary that tolerant strains are capable of producing the desirable toxic metabolites at higher rates, and titers, and not merely tolerate their toxicity [3,4].

The focus of this review: bacterial membranes and cell wall as they relate to metabolite toxicity

The last 10 years have witnessed a large activity in tolerance engineering, including the use of genetic and genomic screens and methods, and synthetic approaches, including focus on the role and impact of membrane transporters[2,4-11]. With few exceptions, the emphasis has been on deleting or overexpressing one gene/protein at a time, where the improvement in tolerance has been small to modest. Little effort has been placed on designing new or modified cellular programs or systems as a means to combat toxicity. Thus, this review focuses on precisely this possibility with emphasis on the understanding and engineering the bacterial membrane and cell wall at the systems level.

Insights and inspiration for engineering tolerance can come from a cell's natural response to toxicity, the differences between a tolerant mutant and the wild type, and even comparing the response mechanisms between strains and species which have varying degrees of naturally occurring tolerance. Beyond comparisons, use of heterologous sources of genetic material, from pure culture or metagenomic sources, can confer tolerance to a toxicity that the host strain may not be able to attain by manipulating only the endogenous genetic material. These are the guiding principles as we navigate through the literature to understand and engineer the tolerance phenotype. 
Native membrane-adaptation mechanisms aim at constant membrane fluidity to combat the impact of toxic environments

Microbial membranes are permeable to small neutral molecules needed for growth (e.g. water, oxygen) as well as waste products for passive expulsion (e.g. carbon dioxide, hydrogen), but create a large free energy barrier to diffusion of large and highly polar/charged molecules (Box 1). In addition to these 'gatekeeper' functions, the ability of the membrane to maintain a chemical potential is crucial for energy generation and other cellular functions. Damage to the cell membrane has long been implicated in the toxicity of various chemicals, with the degree of toxicity correlating to the hydrophobicity[12,13]. Short alcohols and hydrophobic compounds, such as ethanol or butanol, can partition into lipid bilayer membranes[14,15]. These compounds displace water at the membrane/water interface which reduces surface tension. As concentrations and the alcohol carbon chain length rise, the membrane becomes overly fluid, which compromises its integrity[14]. Unsaturated fatty acids allow short alcohols to partition the membrane more than saturated fatty acids, which affects membrane phase behavior[14,16]. Exogenous free fatty acids, common in biodiesel-derived crude glycerol feedstocks, can partition into membranes and have a detrimental effect on growth and fermentation[16-18].

Under changing environmental conditions or stress, such as chemical stress from solvents, acids, and temperature changes, microbes engage several mechanisms for maintaining membrane integrity and fluidity; this is largely done by modifying the fatty acid profile of the phospholipid bilayer, termed the homeoviscous response. Modulating the fatty acid profile is not only important in maintaining membrane stability and integrity, but also for proper function of proteins and cofactors associated with the membrane. Generally, saturated fatty acids increase membrane stability and provide resistance to solvents and high temperatures by packing tightly together due to increasing van der Waals interactions between the acyl chains[19]. In contrast, by breaking up this tightly packed configuration, cis-unsaturated acyl chains increase membrane fluidity [19]. Regarding chain length, longer acyl chain lengths lead to an increase in the stability and rigidity of the membrane while shorter chains increase fluidity.

While the specific responses of microbes to exogenous stress vary, strains naturally tolerant or evolved to be so generally maintain constant membrane fluidity. Exogenous challenge with high levels of hydrophobic chemicals generally leads to an increase in acyl chain tail length and lipid packing density in bacteria, archaea, and yeast[20-24]. Low levels of less hydrophobic compounds (e.g. <10 g/L ethanol) lead to no change or an increase in unsaturated fatty acid content and an increased tail length[20,24]. Of those reported in the literature, $C$. beijerinckii is 
unique in that it completely abolishes production of unsaturated fatty acids under alcohol stress and greatly increases membrane rigidity to enable it to grow in relatively high levels of alcohol[20].

Besides saturated and unsaturated fatty acids, other types of modification can be also engaged for maintaining membrane fluidity. Cyclopropanated and trans-unsaturated fatty acids are discussed below. Additionally, some bacteria do not produce unsaturated fatty acids, but rather produce saturated dimethoxy alkanes (e.g. a strain related to Eubacterium cylindroides) or branched chains (a diverse variety), which have a similar density-reducing effect and decrease in abundance when subjected to solvent stress [23,25]. Strictly anaerobic bacteria (e.g. Clostridium genus) produce plasmalogens, lipids that have a vinyl ether linking group at the $s n$ 1 position of the glycerol as opposed to the ester linking group of a common phospholipid, which are more prevalent during solvent stress[22,25,26]. Very long chain $\alpha, \omega$-dicarboxylic acids, found in certain extremophiles (e.g. Thermoanaerobacter pseudoethanolicus), span the bilayer and are found in greater prevalence during alcohol stress and increase membrane stability[20]. These mechanisms to alter membrane fluidity may prove to be a fruitful target for solvent tolerance engineering.

\section{Adapted and evolved strains confer tolerance by changing lipid profile}

Adaptation and directed evolution are established methods for enabling increased growth in the presence of toxic chemicals or other unfavorable culture conditions. Table 1 lists select literature studies demonstrating changes in lipid composition in evolved strains compared to the parent, which explain in part or in whole the growth-rate increase. Increases in growth rate are generally associated with strains better maintaining optimal membrane fluidity under stress. There is evidence that there are multiple paths to reach a tolerant strain outcome. For example, under octanoic acid stress, short term adaptation of $E$. coli led to an increased unsaturated to saturated lipid ratio (U/S) and an increased acyl chain length as well as a decrease in the cell surface hydrophobicity, while unadapted cells under octanoic acid stress displayed an increase in membrane fluidity and reduced tolerance[27]. An E. coli strain evolved on octanoic acid showed increased unsaturated fatty acid content and produced 5 -fold higher titers of octanoic acid compared to the parent strain[28]. Likewise, an E. coli mutant, EcNR1 G3.2, more tolerant to isobutanol, showed increased U/S during growth in $5 \mathrm{~g} / \mathrm{L}$ isobutanol compared to parent[29]. However, n-butanol evolved $E$. coli strains showed decreased U/S, but still were able to increase the growth rate in $\mathrm{n}$-butanol[30]. So while an increased tolerance to the hydrophobic compound 
is common for the above examples, the mechanism with respect to the lipid profile changes can vary between individual evolved strains.

In contrast to the above $E$. coli findings, evolved Clostridium species consistently produce more saturated acyl chains compared to parent strains under solvent stress. A solvent evolved $C$. beijerinckii strain was shown to have nearly abolished production of unsaturated fatty acids and increased n-butanol production [31], and an ethanol-adapted strain of $C$. thermocellum had a fatty acid profile with a longer average acyl chain length, but no change in branching was observed[25].

\section{Systems-biology analysis confirms that energy metabolism is disrupted by toxic-chemical induced membrane stress}

It has been known that membrane disruption negatively affects the proton gradient and electrochemical potential, and thus the membrane proton motive force[2]. Disrupted proton and ion gradients lead to energetic problems, as the proton motive force generates ATP in aerobic respiration and a minimum adenylate energy charge (a metric of cellular energy availability) is required for viability[32]. Ion gradients, also affected by membrane disruption, are used in active transport processes. Systems biology approaches have enabled greater insight into the genetic, transcriptomic, and metabolomic responses to exogenous stress and the likely mechanisms of tolerance to membrane-disrupting chemicals.

It has been shown through metabolic flux analysis of $E$. coli under octanoic acid stress that carbon flux at the pyruvate node favors the NAD-independent PoxB-catalyzed pyruvate oxidation reaction to acetate, and a ubiquinol $\left(\Delta_{\mathrm{r}} \mathrm{G}^{\circ}=-15 \mathrm{oJJ} / \mathrm{mol}\right)$ instead of the NADdependent pyruvate decarboxylation reaction (aceEF-lpd) leading to acetyl-CoA, carbon dioxide, and $\mathrm{NADH}\left(\Delta_{\mathrm{r}} \mathrm{G}^{\circ}=-35 \mathrm{~kJ} / \mathrm{mol}\right)[33,34]$. The former reaction is more thermodynamically favorable compared to the latter as it produces acetate instead of acetyl-CoA. Acetyl-CoA can enter central metabolism directly or be used to produce an ATP in its conversion to acetate. Consistent with these findings, the transcriptional regulator PdhR, which negatively controls the pyruvate dehydrogenase complex genes (aceEF-lpd) as well as genes in the terminal electron transport chain (ndh and cyoABCDE)[35], has been shown to be upregulated in E. coli undergoing acid stress[36]. Increased expression of PoxB, strictly regulated by RpoS, may relieve oxidative stress in $E$. coli by reducing flux through the pyruvate dehydrogenase complex, which avoids the production of reactive oxygen species by NADH dehydrogenase[37]. This diverted flux reduces the carbon flow through the TCA cycle and reduces NADH production destined for the electron transport chain, increasing relative acetate production and greatly 
reducing biomass[34]. In acetate genomic library selections, increased biomass formation was associated with overexpression of regulated genes in the TCA cycle and biomass production pathways (e.g. amino acid and pyrimidine synthesis)[38].

Isobutanol stress in $E$. coli has been shown to disrupt the function of the membraneembedded electron carrier quinone, which subsequently disrupts cellular respiration and is postulated to be the source of a regulatory response cascade in E. coli[39]. Likewise, changes in expression of the transcription factors ArcA, PdhR, and Fur led to the downregulation of the nuoABCEFGHIKL operon (NADH dehydrogenase I), the cyoABCDE operon (cytochrome $o$ oxidase), and genes associated with the TCA cycle and upregulated the $n d h$ gene (NADH dehydrogenase II)[39]. Wild type $E$. coli under n-butanol stress showed an upregulation of genes related to respiratory functions (nuo, sdh, and cyo operons) and the TCA cycle[40]. Overexpression of membrane-related genes nuoI and pgsA (cardiolipin biosynthesis) increased n-butanol tolerance in E. coli[41]. Transcriptomic analysis of the ethanol tolerant $E$. coli strain LY01, versus its parent KO11 strain, has shown that LYo1 has increased expression of the cyo operon and $n d h$ gene while the nuo operon was down regulated[42]. Genes associated with the aerobic TCA cycle were also generally upregulated in the LYo1 strain; ArcA, part of the quinonedependent ArcAB two-component transcription factor system was expressed higher in the parent KO11 than in the ethanol tolerant LYo1[42]. These studies collectively show the importance of the cellular energetics as a source of toxicity and tolerance in cells under membrane stress.

Engineering of the core lipid metabolism alters the fatty acid profile for engineering tolerance With insight from natural mechanisms of tolerance, including information from naturally solvent tolerant bacteria and evolved mutant strains, engineering the lipid content of the cellular membrane by overexpression or deletion of core lipid synthesis genes has been tested in a variety of contexts.

Overexpression of the $\beta$-hydroxydecanoyl thio-ester dehydrase/isomerase (FabA), which converts 3-hydroxydecanoyl-acyl carrier protein (ACP) to cis-2-decanoyl-ACP, increases the relative amount of saturated fatty acids and increases ethanol tolerance (Fig. 1)[43]. This agrees with results with an $E$. coli genome library-based method which showed $f a b A$ was selected after ethanol selection[44]. Conversely, expression of the fatty acid desaturase gene from B. subtilis, des, decreased the relative amount of saturation and decreased ethanol tolerance[43]. Overexpression of $f a b D$, important as a rate-limiting step in fatty acid biosynthesis, improved growth in n-butanol stress in E. coli by increasing the average acyl chain length and increasing 
$\mathrm{U} / \mathrm{S}[45]$. Deletion of the fatty acid degradation regulator, FadR, which induces expression of fatty acid biosynthesis genes ( $a c c A B C D$, $f a b H D G, f a b B$, and $f a b A$, Fig. 1), increased the saturated fatty acid content compared to the parent and increased hexane tolerance in $E$. coli[46].

\section{Cyclopropane fatty acids are important in membrane homeostasis}

Cyclopropane fatty acids (CFAs) are a substantial portion of the fatty acid content of a variety of bacteria, including E. coli, and are important for membrane lipid homeostasis[19]. CFAs are important to acid resistance in $E$. coli[47]. CFAs have been implicated in solvent tolerance in $P$. putida where c $f a$ (encoding CFA synthase) is under the control of the stress-specific RpoS sigma factor $[48,49]$. The cyclopropanation reaction occurs within the membrane on existing unsaturated fatty acids by the addition of a methylene group from an S-adenosyl-L-methionine (Fig. 1). CFA synthesis does not require ATP or de novo fatty- acid synthesis, making it an attractive target in engineering applications.

Upregulation in cfa was observed in E. coli under octanoic acid, isobutanol and ethanol stress[39,50]. Expression of the cfa gene from a butanol tolerant Enterococcus faecalis-related strain in E. coli resulted in a significant increase in the CFAs and the growth rate of the recombinant strain in both isobutanol and n-butanol[23]. However, either deletion or overexpression of $c f a$ was found to be inhibitory to growth compared to the wild type strain on various octanoic acid concentrations, and both had profound effects on the membrane lipid profile: overexpression of $c f a$ nearly abolished UFAs, and its deletion prevented formation of CFAs [50]. Significant downregulation of cfa was observed in Lactobacillus brevis under nbutanol stress which reduced the CFA content[51].

Transcriptional analysis of several n-butanol-tolerant $E$. coli mutants derived from a combination of n-butanol selection and protoplast-fusion based genome shuffling showed significant upregulation of $c f a$, and $c f a$ overexpression increased the growth rate by $14 \%$ in $0.5 \%$ (v/v) n-butanol[30]. However, a similar study of an isobutanol-tolerant E. coli mutant (EcNR1 G3.2) showed a significant downregulation of $c f a$ and a corresponding reduction in CFA content in the mutant[29]. In contrast, $c f a$ in $C$. acetobutylicum was shown to be upregulated under acid stress or during acidogenesis, but only upregulated at the beginning of n-butanol stress or solventogenesis[52,53]. Overexpression of $c f a$ in $C$. acetobutylicum increased both acid and solvent tolerance, but reduced solvent production, perhaps because a modified membrane perturbed the regulation of solvent production[54]. The differences observed with respect to overexpression of $c f a$ points to subtleties in regulation that are required for proper maintenance 
of membrane homeostasis. While the relationship between CFAs and chemical tolerance is not linear or fully elucidated at this time, it is clearly an area of interest for future work.

\section{Trans-unsaturated fatty acids for modulating membrane density for enhanced tolerance} In some $\mathrm{Gram}^{-}$strains, the periplasmic cis-trans isomerase (Cti) converts unsaturated fatty acids from the cis to the trans conformation as a short term mechanism to maintain membrane fluidity under solvent and temperature stress (Fig. 1)[55]. Cti, not native to E. coli, has been implicated in solvent tolerance by providing a rapid mechanism to increase phospholipid density in the membrane, as the trans-unsaturated fatty acid is more linear in form, akin to a SFA[56]. The cis-trans isomerase gene (cti) from P. aeruginosa was expressed in E. coli and shown to improve tolerance and increase production of octanoic acid by increasing the membrane rigidity[57]; subsequent investigation revealed Cti expression also conferred tolerance to a range of physiological stress conditions including low $\mathrm{pH}$, osmotic pressure, high temperature, and various chemical stressors such as short chain fatty acids, alcohols, organic acids, and aromatic compounds[57].

\section{Phospholipid headgroups as area for continued exploration}

Engineering specific headgroups of glycolipids is a potential exploration area for engineering tolerance as polar headgroups have been shown to be of importance in maintaining membrane homeostasis[58]. For example, C. acetobutylicum under octanol stress modifies the polar headgroups of lipids to stabilize the bilayer by increasing the presence of the charged ethanolamine-phosphate group to certain diglycerides[59]. In a computer simulation, lipid bilayers with phosphatidylcholine (PC, common in eukaryotes, rare in prokaryotes) headgroups were more susceptible to ethanol permeation compared to those with phosphatidylserine (PS) or phosphatidylethanolamine (PE)[6o]. Conversely, knocking out PC synthesis in Acetobacter aceti, an acetic-acid tolerant strain, resulted in an acetic acid sensitive strain while ethanol tolerance was unaffected[61]. Much is left to be understood in how the choice of the polar headgroups could be engineered for improved tolerance and production in metabolic engineering applications.

Beyond the membrane: the cell-wall components, peptidoglycan and lipopolysaccharides act as outer defense barriers

It is common for strains evolved for tolerance to solvents or alcohols to have modifications to the LPS or peptidoglycan, as strengthening these structures can protect the cell from harm. 
An evolved n-butanol-tolerant $E$. coli mutant had differences in expression in many lipopolysaccharide and peptidoglycan biosynthesis genes[30]. Significant changes in expression (mostly increases) of the outer membrane enterobacterial common antigen (ECA) gene cluster ( $r f f H, r f f A, r f f M)$, lipopolysaccharide synthesis and transport (lpcA, $g \operatorname{lm} M, \operatorname{lp} x K, \operatorname{lp} x L, \operatorname{lpt} A)$, and peptidoglycan biosynthesis ( $g \operatorname{lm} M, \operatorname{murE}, \operatorname{murF}, a m i B$ ) were all seen in the n-butanol tolerant mutants[3o]. An isobutanol-tolerant mutant of $E$. coli was found to have an inactivating insertion in the $y h b J$ gene, a negative regulator of the $g \operatorname{lm} S$ gene[62]. Overexpression of the $\mathrm{glmS}$ gene may lead to overproduction of glucosamine-6-phosphate, which is a precursor in both peptidoglycan and LPS synthesis (Fig. 2). The isobutanol-tolerant E. coli strain EcNR1 G3.2 had multiple mutations in lipopolysaccharide (LPS) related genes (i.e. fepE, yjgQ) and upregulation in the $r f a / w a a$ gene cluster, which encodes for LPS biosynthesis (Fig. 2b)[29]. An overall theme of upregulation of genes and gene clusters related to LPS and peptidoglycan biosynthesis is observed in these n-butanol and isobutanol tolerant mutants.

Genomic library selections on various growth inhibitory compounds consistently result in the identification of genes involved in peptidoglycan and LPS biosynthesis. The $\operatorname{lpc} A$ gene, coding the first committed step in the LPS inner core precursor pathway (Fig. 2b), has been selected for using the scalar analysis of library enrichments (SCALES) method against acetate, furfural, and ethanol[38,63,64]. SCALES selection against furfural, a hydrophobic lignocellulosic hydrolysate byproduct known to cause membrane disruption[6], also showed that high copy number of genes related to synthesis of the outer membrane glycolipid ECA (enterobacterial common antigen, $r f f A, r f f C, r f f D, r f f E, r f f G, w z x E$ ) and high copy number of the division and cell-wall synthesis (dcw) gene cluster (murEF-mraY-murD-ftsW-murGC-ddlB$f t s Q$, Fig 2.) resulted in increased fitness[64]. Under ethanol selective pressure, the $2^{\text {nd }}$ highest ranked gene in a SCALES selection was the peptidoglycan maturation gene $\operatorname{mrd} A[44]$, and in an acetate SCALES selection the highest ranking gene was murC[38]. Goodarzi et al. showed that overexpression of peptidoglycan biosynthesis genes (murB, murE, murF, murD, dacA) and a deletion of the peptidoglycan hydrolyzing gene slt increased ethanol tolerance[65], which corresponds well with Nicolaou et al.'s library screening strategy which identified both the murEF-mraY-murD portion of the dcw cluster in a fosmid and the murA-ibaG-mlaBCDEF gene cluster as beneficial for ethanol tolerance [66]. It is interesting to note that many peptidoglycan genes found in the genomic library selections are those that are repressed by PdhR, possibly indicating a regulatory mechanism at work. A strategy for exploring heterologous DNA to find genetic elements conferring product tolerance in $E$. coli has been used to positive effect. A library Lactobacillus plantarum genes randomly inserted into the E. coli KO11 chromosome 
revealed that expression of the heterologous murA2 gene increases ethanol production and tolerance[67].

Unexpectedly, transcriptomic data show reduced peptidoglycan biosynthesis gene expression in ethanol tolerant LYo1 compared to KO11[42]. Diaminopimelate synthesis genes (lysC, asd, dapB, and $d a p D$ ) were downregulated in LYo1 strain, while lysA was upregulated (Fig. 2a)[42]. Transcriptional increases were seen in mep $A$ (murein endopeptidase), and $k d s A$ (CMP-Kdo production, Fig. 2b) while decreases were seen in lpp (major lipoprotein tying the inner membrane to the peptidoglycan, Box 1) and murF in the LYo1 compared to KO11[42]. This stands in contrast to the butanol-tolerant mutants and ethanol genomic library selections discussed above where cell wall synthesis genes were found to be upregulated or have increased fitness.

\section{Recommendations for future direction: a call for a synthetic membrane and cell-wall project}

Analysis of the current literature supports the crucial role of cell membrane and wall in combating chemical toxicity and that innate and engineered changes in their composition suggest them as important engineering targets. While so far only one or a few genes affecting membrane and cell-wall biosynthesis have been engineered, large-scale engineering of these critical cellular components will likely provide better solutions for combating chemical toxicity. Synthetic biology has reached a level of maturity that enables a large-scale engineering of core biosynthetic programs (Figs. 1 \& 2) and the engagement of non-native genes and intermediates that could give rise to robust, functional membrane structures. One could envision the addition of chemical intermediates, whether externally provided or via engineered biosynthesis, that would generate altered membrane and cell-wall structures, from trans-unsaturated fatty acids, novel or enhanced phospholipid headgroups, to dramatically altered core lipid metabolism, enabling extensive changes in the fatty-acid profile. The breadth of the natural repertoire engaged by microbes to deal with chemical toxicity remains largely unexplored, and could guide synthetic biology driven membrane engineering. For example, although it is well known that several Lactobacilli possess unusually high tolerance to solvents and other chemicals[2], the precise nature of it remains a mystery; surely membrane and cell-wall composition are part of the answer for the extraordinary tolerance of these small-genome microbes. Another case study that could guide engineered approaches is that of the alcohol tolerant Zymomonas mobilis. As reviewed[2], its membranes contain over $75 \%$ vaccenic acid (18:1) in polar lipids, while its cytoplasmic membrane contains hopanoids that apparently stabilize the phospholipid bilayer to resist solvent fluidization. These are but a few of the likely large number of unusual membrane 
and cell-wall compositions that have evolved in nature to handle chemical toxicity and such capabilities could be explored through modern metagenomic tools and screens[68,69]. Thus, we call for both such naturally inspired and knowledge-driven $a b$ initio design of cell membranes and walls that would exceed the best of the most tolerant organisms known, e.g., species of Pseudomonas, Zymomonas, Lactobacillus, Rhodococcus, Deinococcus, and Nocardioides[2]. This 'synthome' project could be undertaken through a coordinated, multidisciplinary and multi-team strategy that would aim to holistically attack and resolve the microbial toxicity problem that remains core to the success of microbial processes for the production of chemicals and fuels.

\section{Acknowledgements}

Support for this review from NIH NRSA F32GM109617 and from the NSF CBET- 1511660. We thank Alan Fast for his helpful comments on the manuscript.

\section{Box}

The bacterial membrane and cell wall of peptidoglycan and lipopolysaccharide (for Gram-) provide a first line of defense against exogenous threats to the cell. The cytoplasmic membrane consists of phospholipids from the fatty acid biosynthesis pathway (Fig. 1) with varying degrees of cis-unsaturation which can be modified to cyclopropanated fatty acids or trans-unsaturated fatty acids. Peptidoglycan, or murein, is a polymer network comprising amino acids and sugars formed in lipid II biosynthesis (Fig. 2a) that is bound to the membrane by lipoprotein. The peptidoglycan layer is thick in $\mathrm{Gram}^{+}$bacteria $(>20 \mathrm{~nm})$ compared to Gram- bacteria $(\sim 7 \mathrm{~nm})$, where it is situated in the periplasm. Lipopolysaccharide (LPS) is the outermost feature of Gram- bacteria and consists of the lipid A from the outer membrane and a polysaccharide chain which varies from strain to strain which forms a monolayer on the cell surface (Fig. 2b). While these peptidoglycan and LPS related genes are often implicated in tolerance to various stressors, engineering these structures is an area ripe for exploration. 


\begin{tabular}{|c|c|c|c|c|c|c|c|c|}
\hline $\begin{array}{c}\text { Parent/ } \\
\text { Control Strain } \\
\end{array}$ & $\begin{array}{c}\text { Evolved/ } \\
\text { Engineered strain }\end{array}$ & Condition & $\begin{array}{c}\mathbf{U} / \mathbf{S} \\
\text { Ratio }\end{array}$ & CFA & $\begin{array}{c}\text { Chain } \\
\text { Length } \\
\end{array}$ & Growth & Production & Reference \\
\hline \multirow{5}{*}{$\begin{array}{l}\text { E. coli ML115 } \\
\text { (fadD poxBackA- } \\
\text { pta:cmR) }\end{array}$} & \multirow{5}{*}{ LAR1 (evolved on octanoic acid) } & No Stress & Up & Up & ND & - & Increased C8 & \multirow{5}{*}{ Royce (2015)[28] } \\
\hline & & $30 \mathrm{mM}$ octanoic acid, $\mathrm{pH}$ 7.0 & Up & Slightly Up & Up & Increase & \multirow{4}{*}{ N/A } & \\
\hline & & $430 \mathrm{mM}$ ethanol & ND & Up & ND & - & & \\
\hline & & $30 \operatorname{deg} \mathrm{C}$ & Down & ND & ND & - & & \\
\hline & & $42 \operatorname{deg} C$ & Up & Up & Up & - & & \\
\hline \multirow{2}{*}{ E. coli BW25113 } & MY4 (evolved on n-butanol) & Not stated & Down & Up & - & Increase & $\mathrm{N} / \mathrm{A}$ & \multirow{2}{*}{ Reyes (2012)[30] } \\
\hline & MG6 (evolved on n-butanol) & Not stated & Down & Up & - & Increase & $\mathrm{N} / \mathrm{A}$ & \\
\hline E. coli EcNR1 & G3.2 (evolved on isobutanol) & $0.5 \% \mathrm{w} / \mathrm{v}$ isobutanol $(67.5 \mathrm{mM})$ & Up & Down & - & Increase & N/A & Minty (2011)[29] \\
\hline $\begin{array}{l}\text { C. beijerinckii } \\
\text { ATCC } 10132\end{array}$ & 'solvent adapted' Cbei & Not stated & Down & - & - & Increase & $\begin{array}{l}\text { Increased n- } \\
\text { butanol }\end{array}$ & Isar (2012)[31] \\
\hline \multirow{6}{*}{ E. coli $\mathrm{MG} 1655$} & \multirow{3}{*}{ MG1655 $\Delta c f a$} & No Stress & ND & Zero & ND & Decrease & \multirow{6}{*}{$\mathrm{N} / \mathrm{A}$} & \multirow{6}{*}{ Royce (2014)[5o ] } \\
\hline & & $10 \mathrm{mM}$ octanoic acid & Up & Zero & ND & ND & & \\
\hline & & $20 \mathrm{mM}$ octanoic acid & ND & Zero & ND & ND & & \\
\hline & \multirow{3}{*}{ MG1655+pCA-cfa } & No Stress & ND & Up & ND & Decrease & & \\
\hline & & $10 \mathrm{mM}$ octanoic acid & Down & Up & Down & Decrease & & \\
\hline & & $20 \mathrm{mM}$ octanoic acid & Down & Up & Down & Decrease & & \\
\hline \multirow{5}{*}{$\begin{array}{l}\text { E. coli M1- } \\
\text { Control } \\
\text { (MG1655, ldhA: } \\
\text { FRT-Cat-FRT) }\end{array}$} & \multirow{5}{*}{$\begin{array}{l}\text { M1-12-Pa_cti (plasmid based } \\
\text { P.aeruginosa cti) }\end{array}$} & $10 \mathrm{mM}$ octanoic acid, $\mathrm{pH} 7.0$ & ND & - & ND & Increase & Increased C8 & \multirow{5}{*}{$\operatorname{Tan}(2016)[57]$} \\
\hline & & $343 \mathrm{mM}$ ethanol & - & - & - & Decrease & \multirow{4}{*}{ N/A } & \\
\hline & & 66 mM n-butanol & - & - & - & Increase & & \\
\hline & & $66 \mathrm{mM}$ isobutanol & - & - & - & ND & & \\
\hline & & $42 \operatorname{deg} C$ & - & - & - & Increase & & \\
\hline \multirow{4}{*}{$\begin{array}{l}\text { E. coli } \mathrm{DH}_{5} \mathrm{\alpha}+ \\
\text { pBR322 }\end{array}$} & \multirow{2}{*}{$\begin{array}{l}\mathrm{DH}_{5 \alpha}+\text { pBR-des (B. subtilis } \\
\text { fatty acid desaturase) }\end{array}$} & No Stress & Up & Down & Down & ND & \multirow{4}{*}{ N/A } & \multirow{4}{*}{ Luo (2009)[43] } \\
\hline & & $3 \%$ v/v Ethanol (514 mM) & Up & Down & Up & Decrease & & \\
\hline & \multirow{2}{*}{$\mathrm{DH}_{5} \alpha+$ pBR-fabA $(f a b A)$} & No Stress & Down & Down & Down & ND & & \\
\hline & & $3 \%$ v/v Ethanol (514 mM) & Down & Slightly Up & Down & Increase & & \\
\hline E. coli MDB5 & $\mathrm{MDB}_{5}+\mathrm{pAD} 3(f a b D)$ & 1\% n-butanol (135 mM) & Up & - & Up & Up & $\mathrm{N} / \mathrm{A}$ & Bui (2015)[45] \\
\hline \multirow{2}{*}{$\begin{array}{l}\text { E. coli RLo8 (K- } \\
12 \Delta \text { fadD } \\
\Delta \text { araBAD) }\end{array}$} & RLo8 $\Delta f a b R$ & No Stress & Up & - & - & - & $\mathrm{N} / \mathrm{A}$ & \multirow{2}{*}{$\begin{array}{l}\text { Lennen } \\
\text { (2013)[70] }\end{array}$} \\
\hline & $\mathrm{RLo8}+\mathrm{p} f a b R$ & No Stress & Down & - & - & - & N/A & \\
\hline E. coli BW25113 & JW1176 $(\Delta f a d R)$ & No Stress & Down & Down & Down & - & $\mathrm{N} / \mathrm{A}$ & Oh (2012)[46] \\
\hline
\end{tabular}




\begin{tabular}{|c|c|c|c|c|c|c|c|}
\hline $\begin{array}{c}\text { Parent/ } \\
\text { Control Strain }\end{array}$ & $\begin{array}{c}\text { Evolved/ } \\
\text { Engineered strain }\end{array}$ & Condition & $\begin{array}{c}\text { U/S } \\
\text { Ratio }\end{array}$ & \multicolumn{1}{c|}{ CFA } & $\begin{array}{c}\text { Chain } \\
\text { Length }\end{array}$ & Growth & Production \\
\hline & & $10 \% \mathrm{v} / \mathrm{v} n$-hexane $(760 \mathrm{mM})$ & Down & Up & Down & Increase & $\mathrm{N} / \mathrm{A}$ \\
\hline
\end{tabular}

Table 1. Differences in adaptive responses of evolved or engineered strains compared to the response of the parent strain in the given condition. $\mathrm{U} / \mathrm{S}=$ unsaturated to saturated ratio. $\mathrm{ND}=$ No Difference. - = no data given. N/A = Not Applicable. 


\section{References}

1. Papoutsakis ET: Reassessing the Progress in the Production of Advanced Biofuels in the Current Competitive Environment and Beyond: What Are the Successes and Where Progress Eludes Us and Why. Industrial \& Engineering Chemistry Research 2015, 54:10170-10182.

2. Nicolaou SA, Gaida SM, Papoutsakis ET: A comparative view of metabolite and substrate stress and tolerance in microbial bioprocessing: From biofuels and chemicals, to biocatalysis and bioremediation. Metab Eng 2010, 12:307-331.

3. Reyes LH, Almario MP, Kao KC: Genomic library screens for genes involved in nbutanol tolerance in Escherichia coli. PLoS One 2011, 6:e17678.

4. Zingaro KA, Nicolaou SA, Papoutsakis ET: Dissecting the assays to assess microbial tolerance to toxic chemicals in bioprocessing. Trends in Biotechnology 2013, 31:643653.

5. Cray JA, Stevenson A, Ball P, Bankar SB, Eleutherio ECA, Ezeji TC, Singhal RS, Thevelein JM, Timson DJ, Hallsworth JE: Chaotropicity: a key factor in product tolerance of biofuelproducing microorganisms. Current Opinion in Biotechnology 2015, 33:228-259.

6. Mills TY, Sandoval NR, Gill RT: Cellulosic hydrolysate toxicity and tolerance mechanisms in Escherichia coli. Biotechnol Biofuels 2009, 2:26.

7. Peabody V GL, Winkler J, Kao KC: Tools for developing tolerance to toxic chemicals in microbial systems and perspectives on moving the field forward and into the industrial setting. Current Opinion in Chemical Engineering 2014, 6:9-17.

8. Winkler JD, Erickson K, Choudhury A, Halweg-Edwards AL, Gill RT: Complex systems in metabolic engineering. Current Opinion in Biotechnology 2015, 36:107-114.

9. Kell DB, Swainston N, Pir P, Oliver SG: Membrane transporter engineering in industrial biotechnology and whole cell biocatalysis. Trends in Biotechnology 2015, 33:237-246. 10. Mukhopadhyay A: Tolerance engineering in bacteria for the production of advanced biofuels and chemicals. Trends in Microbiology 2015, 23:498-508.

11. Dunlop MJ: Engineering microbes for tolerance to next-generation biofuels. Biotechnology for Biofuels 2011, 4:32.

12. Zaldivar J, Martinez A, Ingram LO: Effect of alcohol compounds found in hemicellulose hydrolysate on the growth and fermentation of ethanologenic Escherichia coli. Biotechnology and Bioengineering 2000, 68:524-530.

13. Zaldivar J, Martinez A, Ingram LO: Effect of selected aldehydes on the growth and fermentation of ethanologenic Escherichia coli. Biotechnol Bioeng 1999, 65:24-33. 
14. Kurniawan Y, Venkataramanan KP, Scholz C, Bothun GD: n-Butanol partitioning and phase behavior in DPPC/DOPC membranes. $J$ Phys Chem B 2012, 116:5919-5924.

15. Lian J, McKenna R, Rover MR, Nielsen DR, Wen Z, Jarboe LR: Production of biorenewable styrene: utilization of biomass-derived sugars and insights into toxicity. $J$ Ind Microbiol Biotechnol 2016.

16. Bothun GD, Boltz L, Kurniawan Y, Scholz C: Cooperative effects of fatty acids and nbutanol on lipid membrane phase behavior. Colloids Surf B Biointerfaces 2016, 139:6267.

17. Sandoval NR, Venkataramanan KP, Groth TS, Papoutsakis ET: Whole-genome sequence of an evolved Clostridium pasteurianum strain reveals SpooA deficiency responsible for increased butanol production and superior growth. Biotechnol Biofuels 2015, 8:227.

18. Venkataramanan KP, Boatman JJ, Kurniawan Y, Taconi KA, Bothun GD, Scholz C: Impact of impurities in biodiesel-derived crude glycerol on the fermentation by Clostridium pasteurianum ATCC 6013. Appl Microbiol Biotechnol 2012, 93:1325-1335.

19. Zhang YM, Rock CO: Membrane lipid homeostasis in bacteria. Nat Rev Microbiol 2008, 6:222-233.

20. Huffer S, Clark ME, Ning JC, Blanch HW, Clark DS: Role of alcohols in growth, lipid composition, and membrane fluidity of yeasts, bacteria, and archaea. Appl Environ Microbiol 2011, 77:6400-6408.

21. Venkataramanan KP, Kurniawan Y, Boatman JJ, Haynes CH, Taconi KA, Martin L, Bothun GD, Scholz C: Homeoviscous response of Clostridium pasteurianum to butanol toxicity during glycerol fermentation. $J$ Biotechnol 2014, 179:8-14.

${ }^{*}$ 22. Kolek J, Patakova P, Melzoch K, Sigler K, Rezanka T: Changes in Membrane Plasmalogens of Clostridium pasteurianum during Butanol Fermentation as Determined by Lipidomic Analysis. Plos One 2015, 10.

- $\quad$ This study performs lipidomic analysis by high resolution electrospray mass spectrometry in order to show changes in the polar lipid content including those in the plasmalogen form through the fermentation and sporulation cycle. Butanol production and challenge is correlated with plasmalogen abundance.

23. Kanno M, Katayama T, Tamaki H, Mitani Y, Meng XY, Hori T, Narihiro T, Morita N, Hoshino T, Yumoto I, et al.: Isolation of butanol- and isobutanol-tolerant bacteria and 
physiological characterization of their butanol tolerance. Appl Environ Microbiol 2013, 79:6998-7005.

*24. Zu TNK, Athamneh AIM, Wallace RS, Collakova E, Senger RS: Near-Real-Time Analysis of the Phenotypic Responses of Escherichia coli to 1-Butanol Exposure Using Raman Spectroscopy. Journal of Bacteriology 2014, 196:3983-3991.

- Raman spectroscopy was employed in order to study the contents of the E. coli membrane in real time. Experiments showed close alignment between RS measurements and an established GC-FID method.

25. Timmons MD, Knutson BL, Nokes SE, Strobel HJ, Lynn BC: Analysis of composition and structure of Clostridium thermocellum membranes from wild-type and ethanoladapted strains. Appl Microbiol Biotechnol 2009, 82:929-939.

26. Rezanka T, Kresinova Z, Kolouchova I, Sigler K: Lipidomic analysis of bacterial plasmalogens. Folia Microbiol (Praha) 2012, 57:463-472.

27. Royce LA, Liu P, Stebbins MJ, Hanson BC, Jarboe LR: The damaging effects of short chain fatty acids on Escherichia coli membranes. Appl Microbiol Biotechnol 2013, 97:83178327.

**28. Royce LA, Yoon JM, Chen Y, Rickenbach E, Shanks JV, Jarboe LR: Evolution for exogenous octanoic acid tolerance improves carboxylic acid production and membrane integrity. Metab Eng 2015, 29:180-188.

- -*This study shows a comprehensive set of experiments to show how on a physiological level an evolved strain of E. coli outperforms its parent in escaping octanoic acid toxicity. Improved production accompanies tolerance in the mutant, a trait not seen in all evolved strains.

29. Minty JJ, Lesnefsky AA, Lin F, Chen Y, Zaroff TA, Veloso AB, Xie B, McConnell CA, Ward RJ, Schwartz DR, et al.: Evolution combined with genomic study elucidates genetic bases of isobutanol tolerance in Escherichia coli. Microb Cell Fact 2011, 10:18.

30. Reyes LH, Almario MP, Winkler J, Orozco MM, Kao KC: Visualizing evolution in real time to determine the molecular mechanisms of n-butanol tolerance in Escherichia coli. Metab Eng 2012, 14:579-590.

31. Isar J, Rangaswamy V: Improved n-butanol production by solvent tolerant Clostridium beijerinckii. Biomass \& Bioenergy 2012, 37:9-15.

32. Vallon T, Simon O, Rendgen-Heugle B, Frana S, Muckschel B, Broicher A, Siemann-Herzberg M, Pfannenstiel J, Hauer B, Huber A, et al.: Applying systems biology tools to study n- 
butanol degradation in Pseudomonas putida KT2440. Engineering in Life Sciences 2015, 15:760-771.

33. Flamholz A, Noor E, Bar-Even A, Milo R: eQuilibrator--the biochemical thermodynamics calculator. Nucleic Acids Res 2012, 40:D770-775.

**34. Fu YF, Yoon JM, Jarboe L, Shanks JV: Metabolic flux analysis of Escherichia coli MG1655 under octanoic acid (C8) stress. Applied Microbiology and Biotechnology 2015, 99:4397-4408.

- .•This study elucidates the interaction between membrane stress caused by octanoic acid tolerance and changes to metabolism. ${ }^{13} \mathrm{C}$ MFA shows the key role PdhR plays in shunting pyruvate flux away from PDH to PoxB, resulting in reduced biomass accumulation.

35. Ogasawara H, Ishida Y, Yamada K, Yamamoto K, Ishihama A: PdhR (pyruvate dehydrogenase complex regulator) controls the respiratory electron transport system in Escherichia coli. $J$ Bacteriol 2007, 189:5534-5541.

36. King T, Lucchini S, Hinton JC, Gobius K: Transcriptomic analysis of Escherichia coli O157:H7 and K-12 cultures exposed to inorganic and organic acids in stationary phase reveals acidulant- and strain-specific acid tolerance responses. Appl Environ Microbiol 2010, 76:6514-6528.

37. Moreau PL: Diversion of the metabolic flux from pyruvate dehydrogenase to pyruvate oxidase decreases oxidative stress during glucose metabolism in nongrowing Escherichia coli cells incubated under aerobic, phosphate starvation conditions. $J$ Bacteriol 2004, 186:7364-7368.

38. Sandoval NR, Mills TY, Zhang M, Gill RT: Elucidating acetate tolerance in E. coli using a genome-wide approach. Metab Eng 2011, 13:214-224.

39. Brynildsen MP, Liao JC: An integrated network approach identifies the isobutanol response network of Escherichia coli. Mol Syst Biol 2009, 5:277.

40. Rutherford BJ, Dahl RH, Price RE, Szmidt HL, Benke PI, Mukhopadhyay A, Keasling JD: Functional genomic study of exogenous n-butanol stress in Escherichia coli. Appl Environ Microbiol 2010, 76:1935-1945.

41. Reyes LH, Abdelaal AS, Kao KC: Genetic determinants for n-butanol tolerance in evolved Escherichia coli mutants: cross adaptation and antagonistic pleiotropy between n-butanol and other stressors. Appl Environ Microbiol 2013, 79:5313-5320. 42. Gonzalez R, Tao H, Purvis JE, York SW, Shanmugam KT, Ingram LO: Gene array-based identification of changes that contribute to ethanol tolerance in ethanologenic 
Escherichia coli: comparison of KO11 (parent) to LYo1 (resistant mutant). Biotechnol Prog 2003, 19:612-623.

43. Luo LH, Seo PS, Seo JW, Heo SY, Kim DH, Kim CH: Improved ethanol tolerance in Escherichia coli by changing the cellular fatty acids composition through genetic manipulation. Biotechnology Letters 2009, 31:1867-1871.

44. Woodruff LB, Boyle NR, Gill RT: Engineering improved ethanol production in Escherichia coli with a genome-wide approach. Metab Eng 2013, 17:1-11.

**45. Bui LM, Lee JY, Geraldi A, Rahman Z, Lee JH, Kim SC: Improved n-butanol tolerance in Escherichia coli by controlling membrane related functions. Journal of Biotechnology 2015, 204:33-44.

- .• This study systematically overexpresses each $f a b$ gene in E. coli to investigate the differences in butanol tolerance and membrane composition. This study combines multiple membrane engineering strategies simultaneously, making it a good example of a holistic engineering effort.

46. Oh HY, Lee JO, Kim OB: Increase of organic solvent tolerance of Escherichia coli by the deletion of two regulator genes, fadR and marR. Appl Microbiol Biotechnol 2012, 96:1619-1627.

47. Shabala L, Ross T: Cyclopropane fatty acids improve Escherichia coli survival in acidified minimal media by reducing membrane permeability to $\mathrm{H}+$ and enhanced ability to extrude H+. Res Microbiol 2008, 159:458-461.

48. Pini CV, Bernal P, Godoy P, Ramos JL, Segura A: Cyclopropane fatty acids are involved in organic solvent tolerance but not in acid stress resistance in Pseudomonas putida DOT-T1E. Microb Biotechnol 2009, 2:253-261.

49. Pini C, Godoy P, Bernal P, Ramos JL, Segura A: Regulation of the cyclopropane synthase cfaB gene in Pseudomonas putida KT2440. FEMS Microbiol Lett 2011, 321:107-114.

50. Royce LA, Boggess E, Fu Y, Liu P, Shanks JV, Dickerson J, Jarboe LR: Transcriptomic analysis of carboxylic acid challenge in Escherichia coli: beyond membrane damage. PLoS One 2014, 9:e89580.

51. Winkler J, Kao KC: Transcriptional analysis of Lactobacillus brevis to $\mathbf{N}$-butanol and ferulic acid stress responses. PLOS One 2011, 6:e21438.

52. Alsaker KV, Paredes C, Papoutsakis ET: Metabolite stress and tolerance in the production of biofuels and chemicals: gene-expression-based systems analysis of 
butanol, butyrate, and acetate stresses in the anaerobe Clostridium acetobutylicum. Biotechnol Bioeng 2010, 105:1131-1147.

53. Alsaker KV, Papoutsakis ET: Transcriptional program of early sporulation and stationary-phase events in Clostridium acetobutylicum. $J$ Bacteriol 2005, 187:71037118.

54. Zhao Y, Hindorff LA, Chuang A, Monroe-Augustus M, Lyristis M, Harrison ML, Rudolph FB, Bennett GN: Expression of a cloned cyclopropane fatty acid synthase gene reduces solvent formation in Clostridium acetobutylicum ATCC 824. Appl Environ Microbiol 2003, 69:2831-2841.

55. Bernal P, Segura A, Ramos JL: Compensatory role of the cis-trans-isomerase and cardiolipin synthase in the membrane fluidity of Pseudomonas putida DOT-T1E. Environ Microbiol 2007, 9:1658-1664.

56. Junker F, Ramos JL: Involvement of the cis/trans isomerase Cti in solvent resistance of Pseudomonas putida DOT-T1E. J Bacteriol 1999, 181:5693-5700.

${ }^{*} 57$. Tan Z, Yoon JM, Nielsen DR, Shanks JV, Jarboe LR: Membrane engineering via trans unsaturated fatty acids production improves Escherichia coli robustness and production of biorenewables. Metab Eng 2016, 35:105-113.

- -This study shows shows how proper tuning of membrane engineering genes is highly important to the increase in tolerance. The expression of $P$. putida Cti in $E$. coli resulted in increased growth rate under octanoic acid stress.

58. Rilfors L, Lindblom G: Regulation of lipid composition in biological membranes biophysical studies of lipids and lipid synthesizing enzymes. Colloids and Surfaces BBiointerfaces 2002, 26:112-124.

59. Tian B, Guan Z, Goldfine H: An ethanolamine-phosphate modified glycolipid in Clostridium acetobutylicum that responds to membrane stress. Biochim Biophys Acta 2013, 1831:1185-1190.

6o. Konas RM, Daristotle JL, Harbor NB, Klauda JB: Biophysical Changes of Lipid Membranes in the Presence of Ethanol at Varying Concentrations. $J$ Phys Chem $B$ 2015, 119:13134-13141.

61. Hanada T, Kashima Y, Kosugi A, Koizumi Y, Yanagida F, Udaka S: A gene encoding phosphatidylethanolamine $\mathbf{N}$-methyltransferase from Acetobacter aceti and some properties of its disruptant. Biosci Biotechnol Biochem 2001, 65:2741-2748. 
62. Atsumi S, Wu TY, Machado IM, Huang WC, Chen PY, Pellegrini M, Liao JC: Evolution, genomic analysis, and reconstruction of isobutanol tolerance in Escherichia coli. Mol Syst Biol 2010, 6:449.

63. Woodruff LB, Pandhal J, Ow SY, Karimpour-Fard A, Weiss SJ, Wright PC, Gill RT: Genomescale identification and characterization of ethanol tolerance genes in Escherichia coli. Metab Eng 2013, 15:124-133.

64. Glebes TY, Sandoval NR, Reeder PJ, Schilling KD, Zhang M, Gill RT: Genome-Wide Mapping of Furfural Tolerance Genes in Escherichia coli. PLoS One 2014, 9:e87540.

65. Goodarzi H, Bennett BD, Amini S, Reaves ML, Hottes AK, Rabinowitz JD, Tavazoie S: Regulatory and metabolic rewiring during laboratory evolution of ethanol tolerance in E. coli. Mol Syst Biol 2010, 6:378.

66. Nicolaou SA, Gaida SM, Papoutsakis ET: Exploring the combinatorial genomic space in Escherichia coli for ethanol tolerance. Biotechnol $J$ 2012, 7:1337-1345.

**67. Yuan Y, Bi C, Nicolaou SA, Zingaro KA, Ralston M, Papoutsakis ET: Overexpression of the Lactobacillus plantarum peptidoglycan biosynthesis murA2 gene increases the tolerance of Escherichia coli to alcohols and enhances ethanol production. Appl Microbiol Biotechnol 2014, 98:8399-8411.

- •. Looking beyond mutating E. coli, this study explored the Lactobacillus plantarum genomic space for genetic elements conferring ethanol tolerance to an E. coli KO11 host. The clone not only displayed increased tolerance but production as well.

68. Gaida SM, Sandoval NR, Nicolaou SA, Chen YL, Venkataramanan KP, Papoutsakis ET: Expression of heterologous sigma factors enables functional screening of metagenomic and heterologous genomic libraries. Nature Communications 2015, 6.

69. Santero E, Floriano B, Govantes F: Harnessing the power of microbial metabolism. Current Opinion in Microbiology 2016, 31:63-69.

70. Lennen RM, Pfleger BF: Modulating membrane composition alters free fatty acid tolerance in Escherichia coli. PLoS One 2013, 8:e54031. 


\section{Figure captions}

Figure 1. Type II fatty biosynthesis pathway map and unsaturated fatty acid modifications with $E$. coli genes associated with the reactions shown. acc $A B C D$ encodes acetyl-CoA carboxyltransferase, $f a b D$ malonyl-CoA-ACP transacylase, $f a b H \beta$-ketoacyl-ACP synthase III (KASIII), fabG 3-oxoacyl-[acp] reductase, fabZ 3-hydroxy-acyl-[acp] dehydratase, fabI enoyl[acp] reductase, $f a b B$ KASI, fabA $\beta$-hydroxyacyl-[acp] dehydratase/isomerase, fabF KASII, $p l s B$ glycerol-3-phosphate acyltransferase, plsC 1-acylglycerol-3-phosphate O-acyltransferase, $c f a$ cyclopropane fatty acyl phospholipid synthase, and cti cis-trans isomerase. Green arrows represent gene activation and red lines represent repression. 'R' represents any phospholipid head group, [acp] acyl carrier protein, SAM S-adenosyl-L-methionine, and S-adenosyl-Lhomocysteine. Repression by FabR is activated by unsaturated fatty acids.

Figure 2. Cell wall biosynthesis pathways. (A) Peptidoglycan precursor lipid II biosynthesis with $E$. coli genes associated with reactions. pgi encodes phosphoglucose isomerase, glmS Lglutamine:D-fructose-6-phosphate aminotransferase, glm $M$ phosphoglucosamine mutase, glmU N-acetylglucosamine-1-phosphate uridyltransferase, murA UDP-N-acetylglucosamine enolpyruvoyl transferase, murB UDP-N-acetylenolpyruvoylglucosamine reductase, murC UDP$\mathrm{N}$-acetylmuramate-alanine ligase, murI glutamate racemase, murD UDP-N-acetylmuramoyl-Lalanine:D-glutamate ligase, murE UDP-N-acetylmuramoylalanyl-D-glutamate 2,6diaminopimelate ligase, $d a d X / a l r$ alanine racemase, $d d l$ D-alanine-D-alanine ligase, murF UDP-N-acetylmuramoylalanyl-D-glutamyl-2,6-diaminopimelate-D-alanyl-D-alanine ligase (final cytoplasmic step), mraY phospho-N-acetylmuramoyl-pentapeptide transferase, murG Nacetylglucosaminyl transferase, lys $C$ aspartate kinase, asd aspartate semialdehyde dehydrogenase, $\operatorname{dap} A$ dihydrodipicolinate synthase, $\operatorname{dap} B$ tetrahydrodipicolinate succinylase, $\operatorname{serC} / \arg D \mathrm{~N}$-succinyldiaminopimelate aminotransferase, $\operatorname{dap} E \mathrm{~N}$-succinyl-L-diaminopimelate desuccinylase, $d a p F$ diaminopimelate epimerase, and lys $A$ diaminopimelate decarboxylase. Red lines indicate repression by PdhR or lysine. (B) Lipopolysaccharide (LPS) lipid A-core biosynthesis with genes associated with reactions. $\operatorname{lp} x A$ encodes UDP-N-acetylglucosamine acyltransferase, lp $x C$ UDP-3-O-acyl-N-acetylglucosamine deacetylase, lpxD UDP-3-O-(3hydroxymyristoyl)glucosamine $\mathrm{N}$-acetyltransferase, lpxH UDP-2,3-diacylglucosamine hydrolase, $l p x B$ lipid A disaccharide synthase, $l p x K$ tetraacyldisaccharide 4 '-kinase, gutQ/kdsD D-arabinose 5-phosphate isomerase, $k d s A$ KDO-8P synthase, $k d s C$ KDO-8P phosphatase, $k d s B$ KDO cytidylyltransferase, waaA KDO transferase, $l p x L / l p x P$ an acyltransferase, $l p x M$ myristoyl- 
[acp] acyltransferase, lpcA D-sedoheptulose 7-phosphate isomerase, $r f a E$ heptose phosphate kinase/adenyltransferase, gmhB heptose bisphosphate phosphatase, waaC ADP-heptose:LPS heptosyltransferase I (HepI), waaF HepII, waaG LPS glucosyltransferase I, waaP LPS core heptose kinase I (LCHK I), waaQ HepIII, waaY LCHK II, waaB LPS galactosyltransferase, waaO LPS glucosyltransferase II, waa $J$ LPS glucosyltransferase III, and waaU HepIV. Red lines indicate repression by PdhR. 


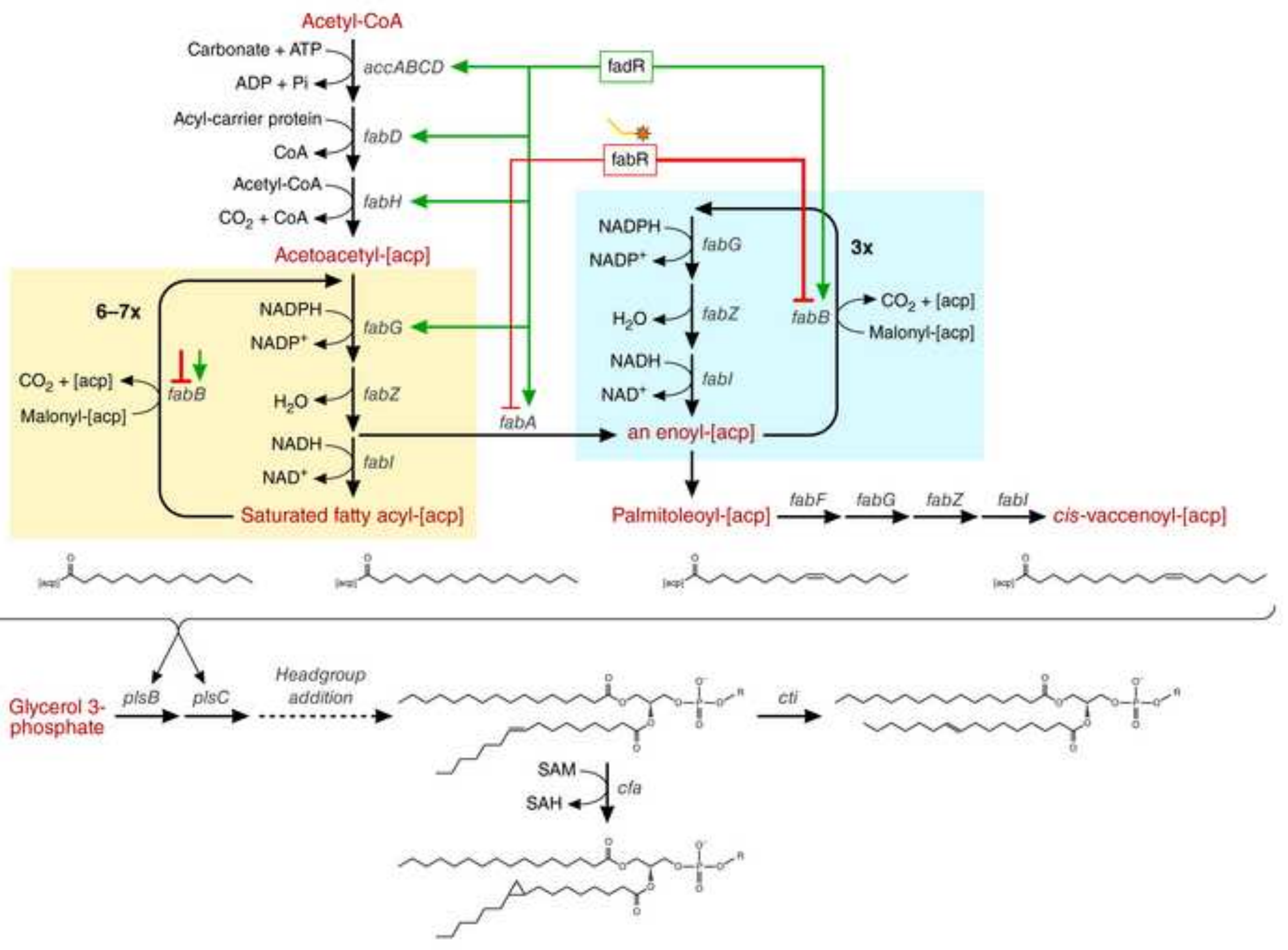


Figure 2

(A)
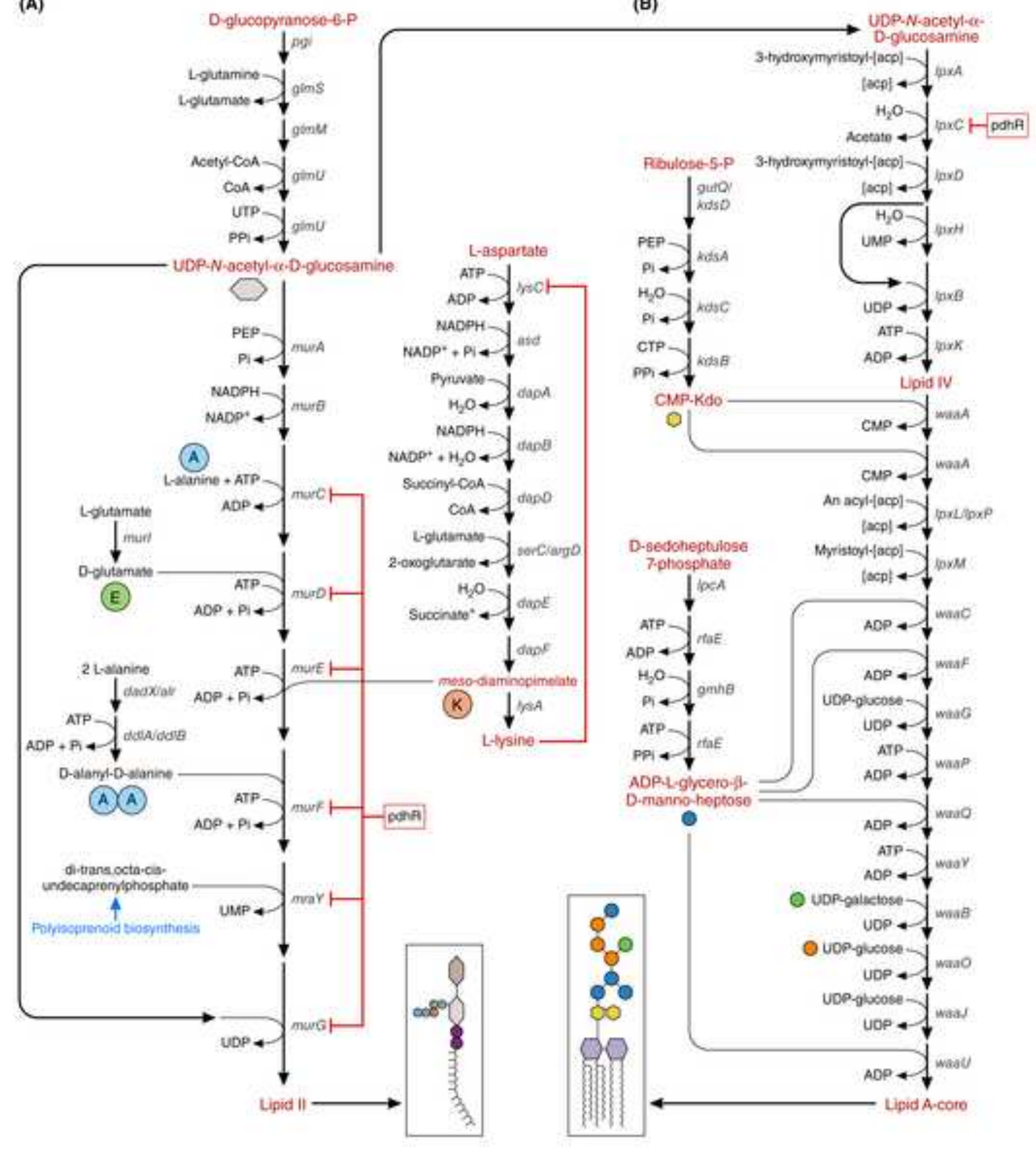

(B) 


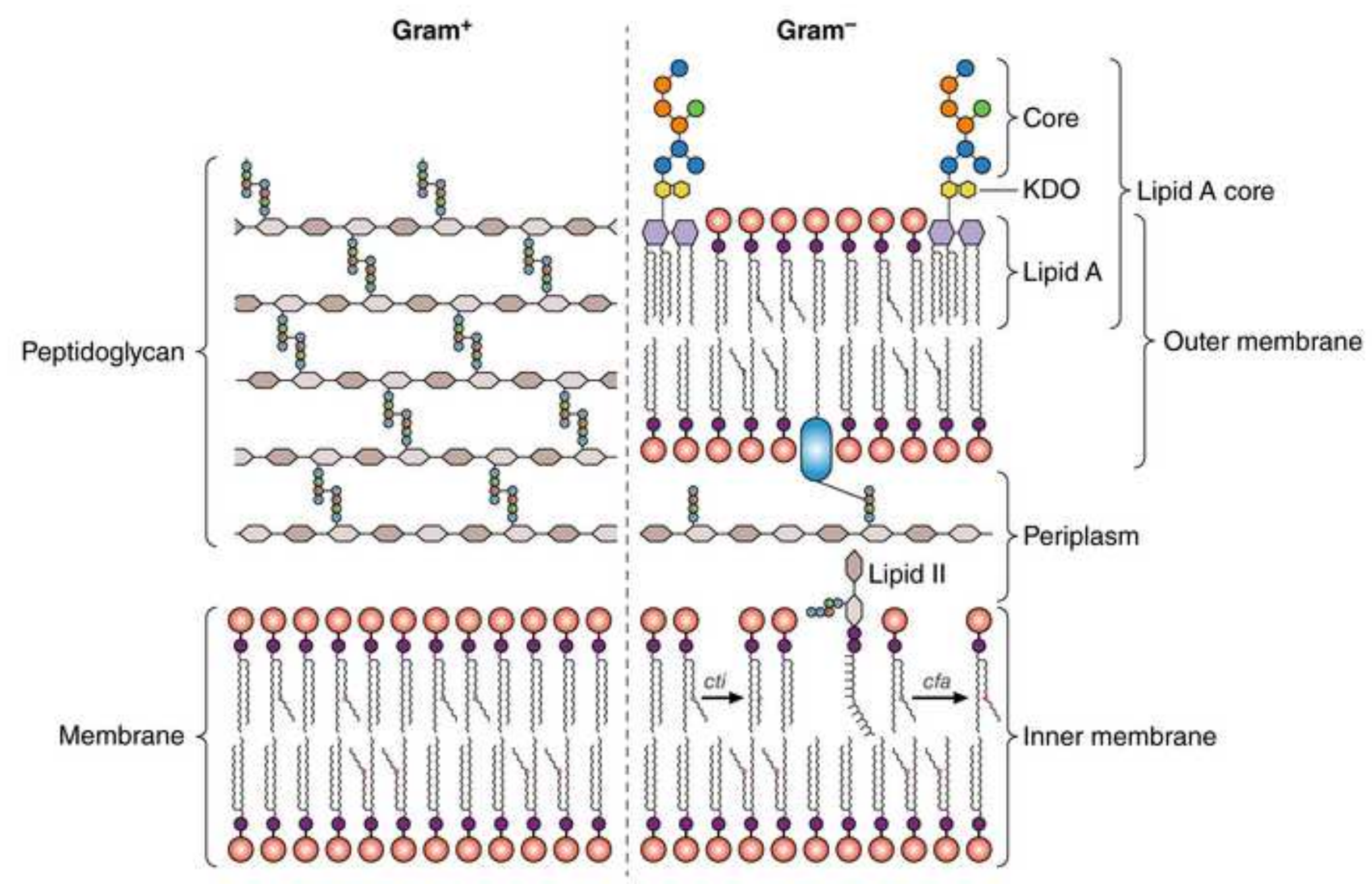




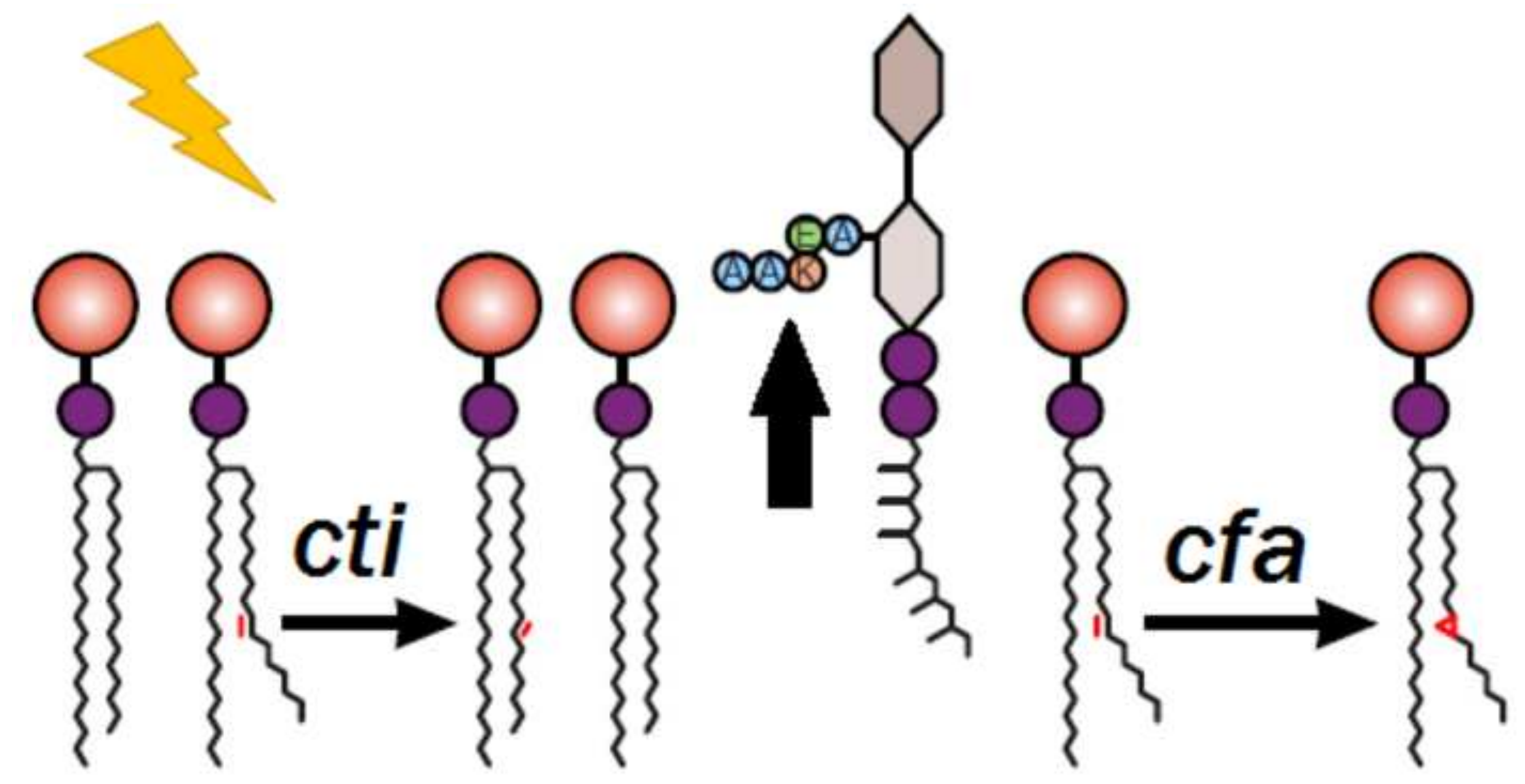

